\title{
Efectividad del plasma rico en fibrina y membrana de colágeno en la regeneración ósea guiada.
}

\section{Effectiveness of fibrin-rich plasma and collagen membrane in guided bone regeneration.}

\author{
Yuliana Príncipe-Delgado ${ }^{1}$, Adrián Mallma-Medina², Yuri Castro-Rodríguez ${ }^{3^{*}}$
}

\section{Cirujana Dentista. Práctica privada.}

2. Cátedra de Patología. Facultad de Odontología.

Universidad Nacional Mayor de San Marcos. Lima. Perú.

3. Docente investigador. Facultad de

Estomatología. Universidad Privada Juan Pablo II. Lima. Perú.

* Correspondencia Autor: Yuri Alejandro Castro Rodríguez | Dirección: Jr. Tomás Catari 463, Urb. El Trébol. Dpto. 201. Los Olivos. | E-mail: yuricastro_16@hotmail.com | Celular 991719062. Trabajo recibido el 17/07/2017.

Aprobado para su publicación el 10/06/2018.

\begin{abstract}
RESUMEN
El objetivo de este estudio fue determinar la efectividad de dos biomateriales, el plasma rico en fibrina (PRF) y la membrana de colágeno en la regeneración ósea guiada. Materiales y método: Fueron utilizados 30 cobayos adultos machos entre 900 a $1100 \mathrm{~g}$. divididos en tres grupos de 10 cobayos cada uno (Grupo A: Control, grupo B: Membrana de colágeno y grupo C: Plasma rico en fibrina). A cada grupo se le creó un defecto óseo mandibular unilateral de $2 \mathrm{~mm}$. Al grupo A no se colocó un biomaterial, en los grupos B y C sí se colocaron los biomateriales en los defectos óseos. A los 15 y 30 días de cicatrización se realizaron cortes histológicos para evaluar la cantidad de fibroblastos, osteocitos y osteoblastos. Resultados: A los 15 días el grupo $C$ formó $40,6 \pm 8,08$ osteocitos/camp y el grupo B $27,6 \pm 4,72(p<0.05)$; a los 30 días el grupo C: $30,6 \pm 11,55$ y el grupo B: $23,6 \pm 3,85$ ( $p>0.05)$. Conclusión: El PRF induce una mayor proliferación celular de forma significativa que los otros grupos en los primeros días de cicatrización; a los 30 días la proliferación es similar con el grupo de membrana de colágeno sin existir diferencias significativas.
\end{abstract}

PALABRAS CLAVE

Regeneración ósea; Fibrina; Colágeno; Cobayas.

Rev. Clin. Periodoncia Implantol. Rehabil. Oral Vol. 12(2); 63-65, 2019.

\begin{abstract}
The aim of this study was to determine the effectiveness of two biomaterials, fibrin-rich plasma (PRF) and collagen membrane in guided bone regeneration. Materials and method: Thirty male guinea pigs were used, weighing between 900 and 1100 g., divided into three groups of 10 guinea pigs each (Group A: control, group B: collagen membrane and group C: fibrin-rich plasma). Each group had a 2-mm unilateral mandibular bone defect. Group A did not receive any biomaterial; in groups B and $C$ the biomaterials were used in the bone defects. After 15 and 30 days of healing, histological sections were performed to evaluate the amount of fibroblasts, osteocytes and osteoblasts. Results: After 15 days, group C formed $40.6 \pm 8.08$ osteocytes / camp and group B $27.6 \pm$ 4.72 ( $p<0.05$ ); After 30 days, group C: $30.6 \pm 11.55$ and group B: $23.6 \pm 3.85$ (p> 0.05 ). Conclusion: During the first 15 days of healing, PRF induces a greater cellular proliferation than the other groups in a significant way; after 30 days, the proliferation is similar to the collagen membrane group without significant differences.
\end{abstract}

KEY WORDS

Bone regeneration; Fibrin; Collagen; Guinea pigs.

Rev. Clin. Periodoncia Implantol. Rehabil. Oral Vol. 12(2); 63-65, 2019.

\section{INTRODUCCIÓN}

La Regeneración Ósea Guiada (ROG) se basa en la formación de nuevo hueso para el relleno de defectos óseos; comprende el uso de membranas con funciones de barrera aptas para evitar la infiltración, en la zona de reparación, de componentes celulares (células epiteliales y conjuntivas) distintos a las células osteopromotoras $^{(1)}$. El tratamiento regenerativo del hueso puede estar basado solamente en la colocación de injertos de hueso autólogo o en combinación con membranas. Elegir una de estas dos alternativas depende de la morfología del defecto óseo ${ }^{(2)}$.

En defectos con paredes óseas conservadas (defecto cerrado) pueden cicatrizar con el uso de hueso autólogo, siempre que el mismo, conjuntamente con el coágulo de sangre permanezca estable dentro del espacio a regenerar y en defectos con ausencia de una o más paredes óseas (defecto abierto), la regeneración puede ser obstaculizada debido a factores como falta de espacio causado por el colapso de tejidos superficiales, o la inestabilidad del coágulo debido a micro-movimientos durante la fase de cicatrización ${ }^{(3,4)}$. En estos casos las membranas además de cumplir con la función de barrera mecánica, cumplen la función de mantenedor de espacio, creando a nivel del defecto óseo un ambiente cerrado delimitado por un lado por las paredes óseas residuales y del otro lado por la membrana que sirve de pared provisional durante el período de cicatrización ${ }^{(5)}$.

Las membranas de colágeno porcino han sido ampliamente utilizadas en los procesos de regeneración ósea, se encuentran conformadas por fibras colágenas del tipo I y III, con escasa capacidad inmunogénica y sin algún componente orgánico o químico(6). Presentan una estructura de doble capa, 
una es compacta y la otra porosa. La capa compacta posee una superficie lisa y condensada que protege contra la infiltración de tejido conectivo, mientras que la capa porosa permite la invasión celular ${ }^{(7)}$. En los últimos años los agentes bioactivos como los concentrados plaquetarios (plasma rico en plaquetas y plasma rico en fibrina) vienen aplicándose como biomateriales capaces de estimular la regeneración ósea principalmente por su elevada concentración de factores de crecimiento ${ }^{(8)}$. El Plasma Rico en Fibrina (PRF) fue definido por Choukroun y col. en Francia y se reconoce como una segunda generación de concentrado de plaquetas ${ }^{(9)}$. Este concentrado exhibe algunas ventajas sobre el plasma rico en plaquetas, entre las cuales se destaca la facilidad de preparación y poco manejo bioquímico de la sangre, lo cual origina una preparación totalmente autóloga ${ }^{(10)}$. En la presente investigación se evaluó la eficacia del plasma rico en fibrina en la regeneración ósea de defectos mandibular en cobayos en comparación con la membrana de colágeno de origen porcino. Hipotetizamos que la efectividad del PRF es superior al de la membrana de colágeno.

\section{MATERIAL Y MÉTODOS}

\section{Diseño y población del estudio}

Estudio experimental que incluyó a 30 cobayos machos de $1000 \pm 100 \mathrm{~g}$ de peso, que fueron ambientados por 7 días en el Bioterio de la Faculta de Farmacia y Bioquímica de la Universidad Nacional Mayor de San Marcos. Lima. Perú. Los cobayos estuvieron a una temperatura ambiente entre $19^{\circ}$ a $22^{\circ} \mathrm{C}$, con una humedad de $40 \%$ a $50 \%$, en períodos de luz/oscuridad de 12 horas alternadas iniciando a las 8 am, con agua y alimentación balanceada ad libitum, acorde a las recomendaciones de la guía ética para animales de experimentación Guide for the Care and Use of Laboratory Animals ${ }^{(11)}$. El tamaño de la muestra se determinó tomando como referencias estudios donde se trabajan con variables y material biológico similar al empleado en esta investigación ${ }^{(12)}$.

\section{Maniobra}

Luego de la semana de ambientación, los cobayos fueron asignados en tres grupos de 10 integrantes cada uno: el grupo A fue el grupo control (no se colocó un biomaterial), el grupo $\mathrm{B}$ se le colocó la membrana de colágeno de origen porcino y el grupo $\mathrm{C}$ se le colocó el plasma rico en fibrina.

\section{Intervención}

Todos los cobayos fueron sometidos a anestesia general con Pentobarbital (Halatal) administrado a dosis de $1 \mathrm{ml} / 2.5 \mathrm{~kg}$ por vía intraperitoneal. Luego se realizó una incisión extraoral de $15 \mathrm{~mm}$ desde el ángulo mandibular derecho hacia adelante. Luego del decolado a espesor total con legra P24g se procedió a realizar la osteotomía con una fresa quirúrgica de carburo de $1 \mathrm{~mm}$ de diámetro. La osteotomía fue extendida hasta crear defectos de forma rectangular de 2x6mm. En 10 cobayos del grupo B se colocó la membrana de colágeno reabsorbible (Matriz de colágeno de origen porcino) ${ }^{*}$ y luego se procedió a la reposición de los tejidos y suturar (puntos simples desde el plano profundo hasta el plano superficial con ácido poliglicólico 5-0 agujas cortantes). En los cobayos del grupo $\mathrm{C}$ se procedió a extraer $3 \mathrm{ml}$ de sangre a través de punción intracardiaca, la sangre fue centrifugada a 3000rpm durante 10 minutost. Se utilizó el kit para la recolección de sangre y centifugación de la marca Xingke $\mathrm{PRF}^{\circledR}$, China (centrífuga preparativa clínica de sobremesa) y tubos de PRF de $10 \mathrm{ml}$ de capacidad de la misma marca.

Luego de la centrifugación, se retiró la capa de glóbulos rojos y el plasma obtenido fue colocada en parrillas metálicas (PRF box, Xingke PRF ${ }^{\circledR}$, China) para la deshidratación del mismo; la membrana obtenida del PRF fue colocada en el defecto óseo para luego suturar por planos similar al grupo B. En los 10 cobayos restantes del grupo A no se colocó biomaterial alguno y se procedió a la reposición de los tejidos y sutura.

\section{Registro $y$ análisis de las variables}

A los 15 días de realizado el procedimiento quirúrgico se seleccionaron de cada grupo cinco cobayos de forma aleatoria, que inmediatamente fueron sacrificados. A los 30 días de realizada la cirugía se sacrificó a los restantes cobayos de cada grupo. La decisión de realizar los cortes histológicos a los 15 y 30 días se basó en el tiempo de cicatrización promedio de los tejidos duros de los animales pequeños (roedores, cobayos y conejos).

Todos los cobayos fueron sacrificados por sobredosis de pentobarbital ( $1 \mathrm{ml} / 250 \mathrm{~g}$ por vía intraperitoneal), luego se procedió a su decapitación y las cabezas fueron desecadas para el posterior análisis histológico de las zonas intervenidas. El análisis histológico estuvo focalizado en el número de osteoblastos y osteocitos para lo cual se prepararon láminas histológicas coloreadas con hematoxilina-eosina. Para la medición celular se procedió a identificar, específicamente, la zona intervenida mediante microscopia; luego, se observó tres campos de dicha zona tomados al azar; esto se realizó en cada una de las láminas obtenidas de cada cuy. De las 3 observaciones se obtuvo la media, la cual fue el número de osteocitos, fibroblastos y osteoblastos para dicho cobayo ${ }^{(12)}$.

\section{Análisis de datos}

Los análisis estadísticos se realizaron con el programa SPSS versión 23. Los resultados se expresan utilizando la media y desviación estándar. La normalidad fue determinada por las pruebas Shapiro-Wilk y Kolmogorov Smirnov. Dada la normalidad de los datos y la homocedasticidad, se utilizó la prueba estadística ANOVA de un factor para determinar si existía alguna diferencia entre los grupos a un nivel de significancia del $95 \%$ y nivel de error del $5 \%$. Se aplicó la prueba de post estimación para comparaciones múltiples HSD Tukey.

\section{RESULTADOS}

Alos 15 días de cicatrización se encontró $72,40 \pm 8.88 \mathrm{cel} / \mathrm{camp}$ (fibroblastos) para el grupo PRF, para el grupo membrana de colágeno un conteo de 31,80 \pm 6.91 y para el grupo control $16,00 \pm 5.57$. Se encontraron diferencias significativas respecto al conteo de fibroblastos, osteoblastos y osteocitos entre los grupos siendo significativo para el grupo PRF $(p<0.05)$ (Tabla 1, Figura 1).

En la segunda evaluación, realizada a los 30 días posteriores al

Tabla 1. Diferencias en los conteos celulares según el biomaterial utilizado a los 15 días de cicatrización.

\begin{tabular}{c|c|c|c|c}
\hline $\begin{array}{c}\text { Tipo de } \\
\text { células }\end{array}$ & $\begin{array}{c}\text { Grupo } \\
\text { Control }\end{array}$ & $\begin{array}{c}\text { Grupo } \\
\text { PRF }\end{array}$ & $\begin{array}{c}\text { Grupo } \\
\text { Membrana } \\
\text { de colágeno }\end{array}$ & $\mathbf{p}$ \\
\hline Fibroblastos & $\begin{array}{c}16,00 \pm 5,57 \\
(9-23)\end{array}$ & $\begin{array}{c}72,40 \pm 8,88 \S \\
(63-87)\end{array}$ & $\begin{array}{c}31,80 \pm 6,91 \\
(23-41)\end{array}$ & $\mathrm{P}<0.05 \ddagger$ \\
\hline Osteoblastos & $\begin{array}{c}28 \pm 6,96 \\
(19-36)\end{array}$ & $\begin{array}{c}69,2 \pm 9,71 \S \\
(61-86)\end{array}$ & $\begin{array}{c}55,6 \pm 2,88 \\
(22-33)\end{array}$ & $\mathrm{P}<0.05 \ddagger$ \\
\hline Osteocitos & $\begin{array}{c}14,6 \pm 4,51 \\
(9-21)\end{array}$ & $\begin{array}{c}40,6 \pm 8,08 \S \\
(31-53)\end{array}$ & $\begin{array}{c}27,6 \pm 4,72 \\
(22-33)\end{array}$ & $\mathrm{P}<0.05 \ddagger$ \\
\hline
\end{tabular}

\section{$\mp$ ANOVA para grupos independientes \\ $\S$ Post hoc HSD de Tukey}
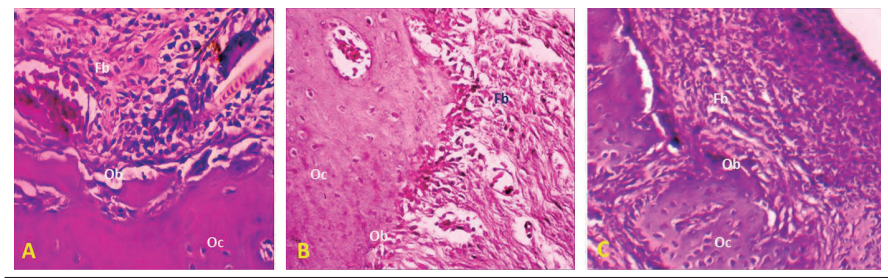

Figura 1. Microfotografía de zonas representativas a los 15 días de cicatrización. Microfotografía con aumento de 400X, coloración HE. A. Control: Escasa cantidad de fibroblastos (Fb), osteoblastos (Ob) y osteocitos (Oc). B. PRF: Se observa abundantes fibroblastos, osteoblastos y osteocitos. C. Membrana de colágeno: Moderada cantidad de fibroblastos, osteoblastos y osteocitos.

procedimiento quirúrgico se encontró $55,6 \pm 11,54 \mathrm{cel} / \mathrm{camp}$ (osteoblastos) para el grupo PRF, para el grupo membrana de colágeno un conteo de 64,6 $\pm 18,77$ y para el grupo control $48 \pm 8,31$. No se encontraron diferencias significativas respecto al conteo de fibroblastos, osteoblastos y osteocitos entre los grupos $(P>0.05)$. (Tabla 2, Figura 2).

\section{DISCUSIÓN}

La Regeneración Ósea Guiada se utiliza de manera exitosa para tratar diversos tipos de defectos óseos a través del uso de membranas con funciones de barrera, como las membranas de colágeno y en los últimos años se prueban otros métodos con mejores resultados clínicos, como el Plasma Rico en Plaquetas (PRP) y el Plasma Rico en Fibrina (PRF); sin embargo, estos buenos resultados clínicos deben contrastarse mediante estudios que determinen desde niveles moleculares, bioquímicos, fisiopatológicos e histológicos, la correspondencia con los beneficios clínicos del uso de éstas técnicas. El presente estudio comparó desde el punto de vista histológico la efectividad de una membrana de colágeno de origen porcino frente al Plasma Rico en Fibrina (PRF) como biomateriales de barrera en el proceso de regeneración ósea guiada.

* La membrana de colágeno de origen porcino (Porciper (C) fue obtenida del banco de tejidos del Instituto de Salud del Niño y Esterilizada en el Instituto Peruano de Energía Nuclear.

† Obtención del plasma rico en fibrina según el protocolo Choukroun. 
Tabla 2. Diferencias en los conteos celulares según el biomaterial utilizado a los 30 días de cicatrización.

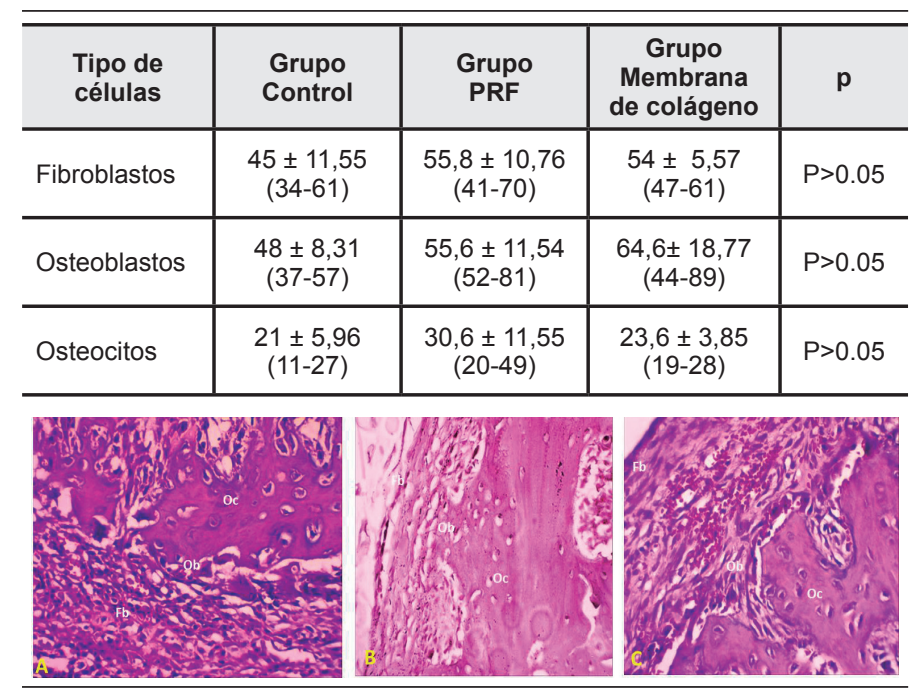

Figura 2. Microfotografía de zonas representativas a los 30 días de cicatrización Microfotografía con aumento de 400X, coloración HE. A. Control: Moderada cantidad de fibroblastos $(\mathrm{Fb})$, osteoblastos $(\mathrm{Ob})$ y osteocitos (Oc) y algunos osteoclastos. B. PRF: Se observa moderada cantidad de fibroblastos, osteoblastos y osteocitos. C. Membrana de colágeno: Se observa moderada cantidad de fibroblastos, osteoblastos y osteocitos y osteoclastos.

Nuestros resultados evidencian una mayor formación de fibroblastos, osteoblastos y osteocitos de forma significativa en el grupo que utilizó PRF a los 15 días de cicatrización. Esto implica una mayor capacidad de osteoinducción del PRF sobre las células óseas que el grupo que tuvo como barrera a la membrana de colágeno de origen porcino. Pola ${ }^{(13)}$ en una evaluación de la reparación ósea de defectos óseos craneales en ratas entre PRF y PRP encuentra similares resultados de formación celular a los 15 días de cicatrización. Acota la función osteoprogenitora de los concentrados plaquetarios sobre las células óseas.

Cabe mencionar que el presente estudio utilizó un modelo experimental de un animal pequeño (cobayo) y que las diferencias en la cicatrización respecto a los animales grandes (incluido el humano) son amplias y se resumen en que: en los cobayos la cicatrización completa de los tejidos duros oscila entre uno a dos meses, el sistema inmunológico es más competente y los procesos infecciosos son mínimos, la tasa de recambio celular y metabolismo es más rápida y los contenidos minerales y de fibras en el tejido óseo difieren respecto a los tejidos humanos; de esta forma, los procesos de cicatrización y regeneración ósea de animales pequeños no pueden ser extrapolados a los tejidos humanos y solo deben utilizarse como referencias del estudio de la biología ósea.

El PRF cuenta con una red de fibrina densa con leucocitos, citoquinas, glicoproteínas estructural y también factores de crecimiento tales como factor de crecimiento derivado de plaquetas, factor de crecimiento endotelial vascular y glicoproteínas tales como trombospondina- 1 ; durante siete días los leucocitos son concentrados en la matriz de PRF y juegan un papel importante en la liberación del factor de la regulación inmune, actividades anti-infecciosas y remodelación de la matriz durante la cicatrización de heridas ${ }^{(14)}$. El lento modo de polimerización de PRF y la capacidad cicatricial crea una arquitectura fisiológica favorable para la herida ${ }^{(15)}$. Al comparar la neoformación celular entre el grupo que utilizó PRF frente a la membrana de colágeno, nuestro estudio no encontró diferencias significativas del conteo celular a los 30 días de cicatrización. Pereira, N. et al ${ }^{(16)}$. encuentra similares resultados a los 30 días al comparar la membrana de colágeno con la agregación de etilcianoacrilato. $Y$ Pallota ${ }^{(17)}$ acota que a los 60 días la reparación ósea en tibias de ratas utilizando PRF no presenta diferencias significativas con los grupos control. Esto puede explicarse debido a que las membranas comienzan su reabsorción a los 30-45 días y el proceso de regeneración ósea depende exclusivamente del propio sistema biológico del organismo. Es de esperar que mientras mayor sea el tiempo de cicatrización los resultados entre el PRF y la membrana de colágeno sean similares y no existan diferencias significativas.

El PRF es un poderoso biomaterial regenerativo utilizado en el tratamiento de defectos intraóseos periodontales, tratamientos de furca y levantamiento de seno. La evidencia científica muestra que el PRF en la regeneración periodontal se utiliza como una membrana para la regeneración tisular guiada que crea un mejor espacio que facilita eventos celulares que son favorables para la regeneración periodontal que conduce a la formación de tejido mineralizado ${ }^{(14)}$. En el caso de la regeneración en defectos óseos de rebordes edéntulos nuestro estudio confirma que su inducción sobre la formación de fibroblastos, osteoblastos y osteocitos es superior al uso de membranas de colágeno durante las dos primeras semanas de posoperatorio.

Sánchez et $a^{(9)}$. en un estudio experimental concluye que la afinidad de los osteoblastos al PRF parece ser superior que la afinidad de los osteoblastos al PRP51.

Thorat et al(18). investigó la eficacia clínica y radiológica de la autotransfusión de PRF en el tratamiento de defectos intraóseos de periodontitis crónica y reportaron una mayor reducción de la profundidad de la bolsa, mayor aumento del nivel de inserción clínica y un mayor relleno del defecto intraóseo en los sitios tratados con PRF que los tratados solo con desbridamiento. De esta forma, el PRF se muestra como un potencial biomaterial que permite la regeneración ósea de defectos óseos periodontales y de rebordes alveolares. Bajo las limitaciones del presente estudio podemos concluir que el plasma rico en fibrina y la membrana de colágeno demostraron ser eficaces como biomateriales en la técnica de regeneración ósea guida; existen diferencias estadísticamente significativas, para la proliferación de fibroblastos, osteoblastos y osteocitos, entre la utilización de PRF y membrana de colágeno a los 15 días, siendo mejor el PRF; sin embargo, a los 30 días no existe diferencias estadísticamente significativas para la proliferación de fibroblastos, osteoblastos y osteocitos entre la utilización de PRF y membrana de colágeno, sin embargo a pesar que la prueba estadística no registra diferencia, según el conteo y análisis microscópico de células el PRF presenta mejor disposición celular que los casos que utilizaron membrana de colágeno.

\section{CONFLICTO DE INTERESES}

Los autores no muestran ningún tipo de conflicto de intereses.

\section{Bibliografía}

1. Trombelli L. Which reconstructive procedures are effective for treating the periodontal intraosseous defect? Periodontol. 2000. 2005;37:88-105.

2. Nyman S, Lindhe J, Karring T, Rylander H. New attachment following surgical treatment of human periodontal disease. J Clin Periodontol. 1982;9:290-296.

3. Castro RY, Bravo FC, Grados SP. Tratamiento regenerativo de las lesiones de furcación, resultados y evidencia científica. Revisión bibliográfica. Rev Dent Chile. 2015;106(2)19-24

4. Lindhe J, Lang N. Periodontología clínica e implantología odontológica. Ed. Médica Panamericana: México, 2001.

5. Rakhmatia YD, Ayukawa Y, Furuhashi A, Koyano K. Current barrier membranes: titanium mesh and other membranes for guided bone regeneration in dental applications. J Prosthodont Res. 2013;57(1):3-14

6. Castro Y, Grados S. Porcine collagen matrix for treating gingival recession. Randomized clinical trial. J Oral Res. 2014;3(1):23-28.

7. Sculean A, Nikolidakis D, Schwarz F. Regeneration of periodontal tissues: combinations of barrier membranes and grafting materials - biological foundation and preclinical evidence. A systematic review. J Clin Periodontol. 2008;35(8):106-116

8. Anitua E. Plasma rich in growth factors: preliminary results of use in the preparation of future site for implant. Int J Oral Maxillofac Implants. 1999;14:529-35.

9. SánchezAR, Sheridan PJ, Kupp LI. Is platelet - rich plasma the perfect enhancement factor? A current review. Int J Oral Maxillofac Implants. 2003;18:93-103.

10. Sunitha V, Munirathnam E. Plasma rico en fibrina. Evolución de una segunda generación de concentrados plasmáticos. Rev Ind J Dent Res. 2008;19:42-46.
11. Guide for the care and use of laboratory animals. Washington D.C.: National Academy Press, 2010.

12. Chumpitaz-Cerrate V, Franco-Quino C, Aguirre-Siancas E, Mallma-Medina A, Rodríguez-Vargas C, Castro-Rodríguez Y. Influencia de la presión de oxígeno ambiental sobre la regeneración ósea guiada. Rev Clin Periodoncia Implantol Rehabil Oral. 2017;10(2):111-114.

13. Pola N. Avaliação do reparo ósseo de defeitos de tamanho crítico tratados com plasma rico em plaquetas ou com fibrina rica em plaquetas. Estudo histomorfométrico e imunoistoquímico em calvárias de ratos. [Tesis doctoral]. UNESP. 2013.

14. Preeja C, Arun S. Platelet-rich fibrin: Its role in periodontal regeneration. Saudi J Dent Res. 2014;5:117-122.

15. Patel RA, Wilson RF, Palmer RM. The effect of smoking on periodontal bone regeneration: A systematic review and meta-analysis. J Periodontol. 2012;83(2):143155.

16. Pereira N S, Santos I.M, Araújo KS. Regeneração óssea guiada utilizando membrana. Rev Bras Odontol. 2012:69(1):39-42.

17. Pallota R. Avaliação do efeito da radiação laser de baixa potência e do plasma rico em fibrina na reparação óssea em ratos. [Tesis doctoral].Universidad de Sao Paulo. 2014

18. Torat M, Prade AR, Pallavi B. Clinical effect of autologous platelet - rich fibrin in the treatment of intra-bone defect: A controlled clinical trial. J Clinic Periodontol. 2011;38 (10):925-32 